\title{
Indian Music Classification using Neural Network based Dragonfly Algorithm
}

\author{
B. Kranthi Kiran \\ Associate Professor \\ Computer Science \& Engineering \\ JNTUH College of Engineering, Hyderabad \\ kranthikirankiran794@gmail.com
}

\begin{abstract}
Generally, Indian Classical Music (ICM) is categorized into 2 Hindustani and Carnatic. Although, aforesaid music formats possess the same base, the presentation manner is different in numerous ways. The ICM basic modules are taala and raga. Fundamentally, Taala indicates rhythmic beats else patterns. From the flow of swaras, the raga is ascertained that is indicated as extensive terms. On the basis of few important factors, the raga is indicated namely aarohana-avarohna and swaras and distinctive phrases. In practice, the basic frequency is Swara that is exact via time period. In addition, there are numerous other issues with the automatic raga identification technique. Hence, raga is identified in this research without using precise note series information and vital to use an effectual classification technique. This research develops an effectual raga recognition model via the Carnatic genre music that is efficiently identified for the data mining models, which is also included. Here, the Neural Network (NN) model is proposed which is an adaptive classifier in that the feature set is exploited to learning, and the data mining models are used for the classification techniques. Moreover, a meta-heuristic approach is used for the adaptive classifier to obtain the extracted feature set knowledge. As the learning technique plays an important role in describing the accuracy of the raga identification model, it prefers to adopt the Dragonfly algorithm.
\end{abstract}

Keywords: Classification, ICM, NN, Raaga, Swaras.

\begin{tabular}{ll} 
Nomenclature & \\
\hline STFT & \multicolumn{1}{c}{ Short-Time Fourier Transform } \\
\hline ICM & Indian Classical Music \\
MFCCs & Mel-frequency cepstral coefficients \\
MIR & Music information Retrieval \\
ML & Machine Learning \\
GA & Genetic Algorithm \\
KNN & K-Nearest Neighbors \\
CM & Carnatic Music \\
SWIPE & Sawtooth Waveform Inspired Pitch Estimator \\
FT & Fourier transform \\
ERBs & Equivalent Rectangular Bandwidth \\
LPCCs & Linear Prediction Cepstral Coefficients \\
SVM & Support Vector Machine \\
KKT & Kosambi-Karhunen-Loeve transform \\
FDLP & Frequency Domain Linear Pre Iction \\
\hline
\end{tabular}

\section{Introduction}

MIR is a science of information retrieval regarding music, both dynamically and statically [1]. Music represents is an art that is enthused and exploited by each person, many research is carried out in this areas since the 1990s. MIR is represented as a maximum learning curve and it is ahead of text information knowledge retrieval. Music is a topic in itself; hence it needs a strong comprehension of 
analytical, theoretical and convenient effects [2]. In numerous manners, the music varies from the text. One of the major significant realities is that music is multifaceted, hence studies in this area are somewhat is difficult. The two important methods, MIR is tackled based on the content and the metadata. At first, most of the studies were done on indexing of music, music classification which is on the basis of the artist, compositions and other static information which included text-based retrieval. In musical content retrieval, the content-based MIR is concentrated namely melody, pitch and rhythm which is exploited to extract the audio features to identify and classify the songs [3].

The speedy growth of digital technologies, multimedia industry, internet, and has motivated an enormous information overload and an obligation of effectual information filtering systems and specific suggestion systems. Present significant internet stores comprise millions of tracks in the digital music industry scenario that make difficult search, retrieval and identification of music appropriate for one user [4]. The music feature is categorized into 3 classifications such as high level, low level, and medium level features. For the genre of the song classification recommendations, aforesaid features are exploited, classifications on the basis of the textual features so on, the procedure of aforesaid classifications may not present the finest outcomes. Therefore, the optimal set involves features from all the 3 classifications on the basis of the feature variance with cost, time and a waveform. The restricted objective of the feature ranges chosen via domain knowledge can be extensive by exploiting the feature selection techniques in ML [12].

In numerous manners, the music or speech classification task is performed by exploiting diverse classifiers and features. In some works, the speech-detailed features for music or speech classification were performed, which was motivated by the music exploit detailed features. Moreover, there are numerous motivations to look at this task from a speech particular perspective. The signal of music cannot be explored without trouble because of the attendance of various kinds of music sources. Therefore, robust feature selection concerning the music is a complex task [5]. Speech is generated by humans and widespread research was performed to research the production of speech and opinion systems regarding the vocal tract system, source of excitation, and the dynamics related with them. To generate a specific sound unit, the shape of glottal vibration, and vocal tract, is considered as the excitation source remnants mainly similar for a specific speaker. Although there are numerous factors such as basic pronunciation, frequency and individual anatomy which control the production of a specific sound over various speakers, the most important factors included in the production are vocal tract shape. Raga identification for humans is a tedious issue, and it takes years to obtain recognition skill for a huge corpus. They are highly distracted from the actual melodic series that recognizing in performances.

The main contribution of this research is to work on an Neural Network classifier to classify the ragas and the Dragonfly technique is used to train the NN model. Finally, the developed method is evaluated with existing techniques and exhibits the betterment of the proposed technique.

\section{Literature review}

In 2020, M.S. Sinith et al [1], worked on the ICM, which was an attractive relative among Just Intonation and Fibonacci series. Furthermore, by exploiting the Fibonacci series, the discrete model of pitch contours on the basis of a table was derived for the ICM. To indicate the melody, aforesaid pitch contours were efficiently exploited, and also, it acts as a tool for the retrieval of music information. Moreover, a pitch distribution based on the Fibonacci series was developed. In 2017, Prafulla Kalapatapu et al [2], presented the impact of four feature selection techniques such as GA, information gain, Forward feature selection. Additionally, the correlation on the basis of 4 diverse classifiers such as Decision tree C4.5, KNN, NN, and SVM. From music segments, feature vectors were extracted from the initial thirty secs and finally 30 secs of the music signal. In 2017, Banriskhem K. Khonglah et al [3], developed speech-detailed features for music or speech classification. Features indicating vocal tract system and the syllabic rate of speech, excitation source, were discovered. By exploiting threshold-based technique aforesaid features were nonlinearly mapped and integrated to carry out the classification task. Furthermore, speech-specific feature performance was calculated by exploiting the classifiers namely SVM and Gaussian mixture models. In 2018, Snehlata Barde and Veena Kaimal [4] studied signal processing via Praat as the basic model. They had also worked on the classification of the raga in CM. Moreover, they recognized superior choices of classification and recognition of ragas. Further, by exploiting digital speech or signal processing numerous current studies in MIR models were presented for dynamically retrieving information. At last, the developed method presents a simple technique for the ragas classification. In 2018, Y.V. Srinivasa Murthy et al [5], worked on the MIR mode, to aid in tagging every segment of an audio clip. Here, an attempt was done for the non-vocal and vocal regions segmentation by exploiting new features. Here, the features like LPCCs, MFCCs, FDLP values, were considered. 


\section{Evaluation of Raga Audio Signal}

\subsection{STFT Feature}

Consider denoised raga audio signal as y(n). In STFT [6], the time-frequency content of y(n) is classified by exploiting the time-frequency dissemination model. The time-frequency illustration should fulfill property called 'magnitude wise shift-invariance property'. The particular property declaration is stated as below:

Time-frequency dissemination is indicated as $\mathrm{T}_{\mathrm{z}}(1, \mathrm{~m})$. It is represented as magnitude-wise shiftinvariant, which is indicated in eq. (1), if $\mathrm{z}_{\mathrm{t}}(1)=\mathrm{z}\left(1-\mathrm{l}_{\mathrm{t}}\right) \cdot \mathrm{e}^{\mathrm{j} 2 \Pi \mathrm{m}_{\mathrm{t}} \mathrm{l}}$.

$$
\left|\mathrm{T}_{\mathrm{z}_{\mathrm{t}}}(\mathrm{l}, \mathrm{m})\right|=\left|\mathrm{T}_{\mathrm{z}}\left(\mathrm{l}-\mathrm{l}_{\mathrm{t}}, \mathrm{m}-\mathrm{m}_{\mathrm{t}}\right)\right| ; \forall \mathrm{z}(\mathrm{l}), \mathrm{l}_{\mathrm{t}}, \mathrm{m}_{\mathrm{t}}
$$

Eq. (2) represents the kernel-based linear time-frequency dissemination $\mathrm{T}_{\mathrm{Z}}(1, \mathrm{~m})$.

$$
\mathrm{T}_{\mathrm{z}}(\mathrm{l}, \mathrm{m})=\int \mathrm{K}\left(\mathrm{l}, \mathrm{m}, \mathrm{l}^{\prime}\right) \mathrm{z}\left(\mathrm{l}^{\prime}\right) \mathrm{d} \mathrm{l}^{\prime}
$$

$\mathrm{K}\left(1, \mathrm{~m}, \mathrm{l}^{\prime}\right)$ indicates dissemination kernel, Eq. (3), magnitude-wise shift-invariance in time, which requires a model.

$$
\mathrm{T}_{\mathrm{z}}(1, \mathrm{~m})=\mathrm{e}^{\mathrm{j} \hat{\Psi}(\mathrm{l}, \mathrm{m})} \int \mathrm{K}\left(\mathrm{l}-\mathrm{l}^{\prime}, \mathrm{v}\right) \mathrm{z}\left(\mathrm{l}^{\prime}\right) \mathrm{dl} \mathrm{l}^{\prime}
$$

As there is a nearby association between energy distribution of magnitude and time-frequency distribution signal, e $\mathrm{e}^{\mathrm{j}} \hat{\Psi}(1, \mathrm{~m})$ is deleted in residual derivations. The magnitude inferences wise shiftinvariance with frequency is identified and indicated in Eq. (4)

$$
\begin{aligned}
\mathrm{T}_{\mathrm{X}}(\mathrm{l}, \mathrm{m}) & =\int \mathrm{K}\left(\mathrm{l}-\mathrm{l}^{\prime}, \mathrm{m}\right) \int \mathrm{Z}\left(\mathrm{m}^{\prime}\right) \mathrm{e}^{\mathrm{j} 2 \Pi \mathrm{v}^{\prime} \mathrm{u}^{\prime}} \mathrm{dm^{ \prime }} \mathrm{dl}^{\prime} \\
& =\mathrm{e}^{\mathrm{j} 2 \Pi v \mathrm{vu}} \int \Omega\left(1, \mathrm{l}^{\prime}\right) \mathrm{e}^{-\mathrm{j} 2 \Pi\left(\mathrm{m}-\mathrm{m}^{\prime}\right) \mathrm{u}} \mathrm{Z}\left(\mathrm{m}^{\prime}\right) \mathrm{dm^{ \prime }}
\end{aligned}
$$

whereas $\mathrm{Z}(\mathrm{m})$ indicates FT of $\mathrm{z}(\mathrm{l})$ as well as $\Omega\left(\mathrm{m}-\mathrm{m}^{\prime}\right)=\int \mathrm{K}\left(\mathrm{l}^{\prime \prime}, \mathrm{m}\right) \mathrm{e}^{-\mathrm{j} 2 \Pi l \mathrm{~m}} \mathrm{dl} \mathrm{l}^{\prime \prime}$. Eq. (5) indicates magnitudewise shift-invariance regarding frequency. Therefore, the kernel is indicated as in Eq. (6).

$$
\begin{aligned}
\Omega\left(m, m^{\prime}\right) & =H\left(m-m^{\prime}\right) e^{j \bar{\psi}(m)} \\
\mathrm{K}\left(1, m^{\prime}\right) & =e^{j \bar{\psi}(m)} \int \mathrm{H}\left(\mathrm{m}-\mathrm{m}^{\prime}\right) \mathrm{e}^{\mathrm{j} 2 \Pi \mathrm{m}^{\prime} l} \mathrm{dm}^{\prime} \\
= & \mathrm{h}(-\mathrm{l}) \mathrm{e}^{\mathrm{j} 2 \Pi l \mathrm{~m}} \mathrm{e}^{\mathrm{j} \bar{\psi}(\mathrm{m})}
\end{aligned}
$$

As stated in eq. (7) $\mathrm{e}^{\mathrm{j} 2 \Pi v \mathrm{vml}}$ and $\mathrm{e}^{\mathrm{j} \bar{\psi}(\mathrm{m})}$ phase could be disregarded, magnitude-wise shift-invariant timefrequency dissemination. This is the same as STFT form with $\mathrm{h}(\mathrm{u})$ the kernel that is stated as Eq. (8).

$$
\begin{aligned}
& \mathrm{T}_{\mathrm{z}}(\mathrm{l}, \mathrm{m})=\int \mathrm{h}\left(\mathrm{l}^{\prime}-\mathrm{l}\right) \mathrm{z}\left(\mathrm{l}^{\prime}\right) \mathrm{e}^{-\mathrm{j} 2 \Pi m l^{\prime}} \mathrm{dl}^{\prime} \\
& \operatorname{STFT}_{\mathrm{f}^{\prime}}(\mathrm{n})=\mathrm{T}_{\mathrm{z}}(1, \mathrm{~m})
\end{aligned}
$$

\subsection{NMF Feature}

Let non-negative data vector of $\mathrm{y}(\mathrm{n})$ as $\mathrm{A} \in \mathrm{R}^{1 \times \mathrm{x}} ; \mathbf{1 \times x}$ is $1 \mathrm{D}$ samples. The NMF [7] objective is to ascertain two nonnegative matrices that are indicated as Eq. (9) and (10), whereas $s$ indicated as dimensional space.

$$
\begin{gathered}
E \in \mathrm{R}^{1 \times \mathrm{s}} \\
\mathrm{F} \in \mathrm{R}^{1 \times \mathrm{s}}
\end{gathered}
$$

The non-negative constraints obtain a part-based indication as they consent merely augmented and not either subtractive or integrations. The renowned cost functions are stated as below:

a) The square Euclidean distance among EF and A is stated in Eq. (11), whereas ||$\cdot||_{M}$ indicates matrix Frobenius norm.

$$
|| \mathrm{A}-\mathrm{EF}||_{\mathrm{M}}=\sum_{\mathrm{K}-1}^{\mathrm{x}}|| \mathrm{A}_{\mathrm{K}}-(\mathrm{EF})_{\mathrm{K}}||^{2}
$$

b) Eq. (12) indicates comprehensive Kullback-Leibler divergence $V$ among A and EF .

$$
\mathrm{V}(\mathrm{A}|| \mathrm{EF})=\sum_{\mathrm{K}-1}^{\mathrm{x}} \mathrm{A}_{\mathrm{K}} \log \frac{\mathrm{A}_{\mathrm{K}}}{(\mathrm{EF})_{\mathrm{K}}}-\mathrm{A}_{\mathrm{K}}+(\mathrm{EF})_{\mathrm{K}}
$$

Using multiplicative technique [9] both the cost functions are solved. Eq. (13) and (14) indicates for square Euclidean distance update imperative of non-negative matrices $\mathrm{E}_{\mathrm{K}}$ and $\mathrm{F}_{\mathrm{K}}$. Eq. (15) and (16) states the update regulation for the variance of $\mathrm{E}_{\mathrm{K}}$ and $\mathrm{F}_{\mathrm{K}}$, whereas $\mathrm{K}=1, \ldots . \mathrm{x}$. Eq. (17) indicates the $\mathrm{NMF}$ feature $\mathrm{NMF}_{\mathrm{f}^{\prime}}(\mathrm{n})$. 


$$
\begin{aligned}
& \mathrm{F}_{\mathrm{K}} \leftarrow \frac{\left(\mathrm{E}^{\mathrm{T}} \mathrm{A}\right)_{\mathrm{K}}}{\left(\mathrm{E}^{\mathrm{T}} \mathrm{EF}\right)_{\mathrm{K}}} \mathrm{F}_{\mathrm{K}} \\
& \mathrm{E}_{\mathrm{K}} \leftarrow \frac{\left(\mathrm{AF}^{\mathrm{T}}\right)}{\left(\mathrm{EFF}^{\mathrm{T}}\right)} \mathrm{F}_{\mathrm{K}} \\
& \mathrm{F}_{\mathrm{K}} \leftarrow \frac{\sum_{\mathrm{l}} \mathrm{E}_{\mathrm{l}} \mathrm{A}_{\mathrm{lK}} /(\mathrm{EF})_{l \mathrm{~K}}}{\sum_{\mathrm{l}} \mathrm{E}_{\mathrm{l}}} \mathrm{F}_{\mathrm{K}} \\
& \mathrm{E}_{\mathrm{K}} \leftarrow \frac{\sum_{\mathrm{l}} \mathrm{E}_{\mathrm{Kl}} \mathrm{A}_{\mathrm{K}} /(\mathrm{EF})_{l \mathrm{~K}}}{\sum_{l} \mathrm{~F}_{\mathrm{Kl}}} \mathrm{E}_{\mathrm{K}} \\
& \mathrm{NMF}_{\mathrm{f}^{\prime}}(\mathrm{n})=\left(\mathrm{E}_{\mathrm{K}}, \mathrm{F}_{\mathrm{K}}\right)
\end{aligned}
$$

\subsection{Pitch Feature}

The SWIPE pitch assessment [8] is stated as below:

Eq. (18) denotes the SWIPE estimation of the pitch $\operatorname{Pitch}_{\mathrm{f}^{\prime}}(\mathrm{n})$ at a time $\mathrm{t}$ is estimated.

$$
\operatorname{Pitch}_{\mathrm{f}}(\mathrm{t})=\underset{\mathrm{f}}{\arg \max } \frac{\int_{0}^{\operatorname{ERB}\left(\mathrm{f}_{\max }\right)} \frac{1}{\eta(\varepsilon)} \hat{\mathrm{K}}(\mathrm{f}, \eta(\varepsilon))|\tilde{\mathrm{X}}(\mathrm{t}, \mathrm{f}, \eta(\varepsilon))|^{1 / 2} \tilde{\mathrm{d}} \varepsilon}{\left(\int_{0}^{\operatorname{ERB}\left(\mathrm{f}_{\max }\right)} \frac{1}{\eta(\varepsilon)}\left[\hat{\mathrm{K}}^{+}(\mathrm{f}, \eta(\varepsilon))\right]^{2} \tilde{\mathrm{d}} \varepsilon\right)^{1 / 2}\left(\int_{0}^{\operatorname{ERB}\left(\mathrm{f}_{\max }\right)}|\widetilde{\mathrm{X}}(\mathrm{t}, \mathrm{f}, \eta(\varepsilon))| \tilde{\mathrm{d}} \varepsilon\right)^{1 / 2}}
$$

whereas;

$$
\begin{aligned}
& \hat{\mathrm{K}}\left(\mathrm{f}, \mathrm{f}^{\prime}\right)=\left\{\begin{array}{lc}
\cos \left(2 \pi \mathrm{f}^{\prime} / \mathrm{f}\right) & \text { if } 3 / 4<\mathrm{f}^{\prime} / \mathrm{f}<\mathrm{n}(\mathrm{f})+1 / 4 \\
\frac{1}{2} \cos \left(2 \pi \mathrm{f}^{\prime} / \mathrm{f}\right) & \text { if } 1 / 4<\mathrm{f}^{\prime} / \mathrm{f}<3 / 4 \operatorname{orn}(\mathrm{f})+1 / 4<\mathrm{f}^{\prime} / \mathrm{f}<\mathrm{n}(\mathrm{f})+3 / 4 \\
0 & \text { otherwise }
\end{array}\right. \\
& \tilde{\mathrm{X}}\left(\mathrm{t}, \mathrm{f}, \mathrm{f}^{\prime}\right)=\int_{-\infty}^{\infty} \mathrm{wi}_{4 \mathrm{k} / \mathrm{f}}\left(\mathrm{t}^{\prime}-\mathrm{t}\right) \mathrm{k}\left(\mathrm{t}^{\prime}\right) \mathrm{e}^{-\mathrm{j} 2 \pi \mathrm{f}^{\prime} \mathrm{t}^{\prime}} \tilde{\mathrm{dt}} \mathrm{t}^{\prime}
\end{aligned}
$$

$\eta(\cdot)$ transforms frequency from ERBs into Hertz, $\varepsilon$ denotes the frequency in ERBs, ERB(.) transforms frequency from Hertz into ERBs, $\hat{\mathrm{K}}^{+}\left(\right.$.) indicates a positive segment of $\hat{\mathrm{K}}(\cdot), \mathrm{n}(\mathrm{f})=\left\lfloor\mathrm{f}_{\max } / \mathrm{f}-/ 4\right\rfloor \mathrm{and} \mathrm{wi}_{4 \mathrm{k} / \mathrm{f}}(\mathrm{t})$ indicates window function with size $4 \mathrm{k} / \mathrm{f}$ which is stated in eq. (20).

\section{Developed Raga Recognition Technique}

Fig 1 demonstrates the developed raga recognition technique. Here, feature extraction is the primary process that consequences in features such as NMF feature $\mathrm{NMF}_{\mathrm{f}^{\prime}}(\mathrm{n})$, STFT feature $\mathrm{STFT}_{\mathrm{f}^{\prime}}(\mathrm{n})$, and SWIPE feature $\operatorname{Pitch}_{\mathrm{f}^{\prime}}(\mathrm{n})$.

Moreover, to minimize dimensions PCA is exploited for obtained features. The consequences dimensional minimized features are fed to the input to classification procedure. Furthermore, an optimized weight is multiplied by the input features. Therefore, the relation between the features is maximized, hence precise recognition can be shown. 


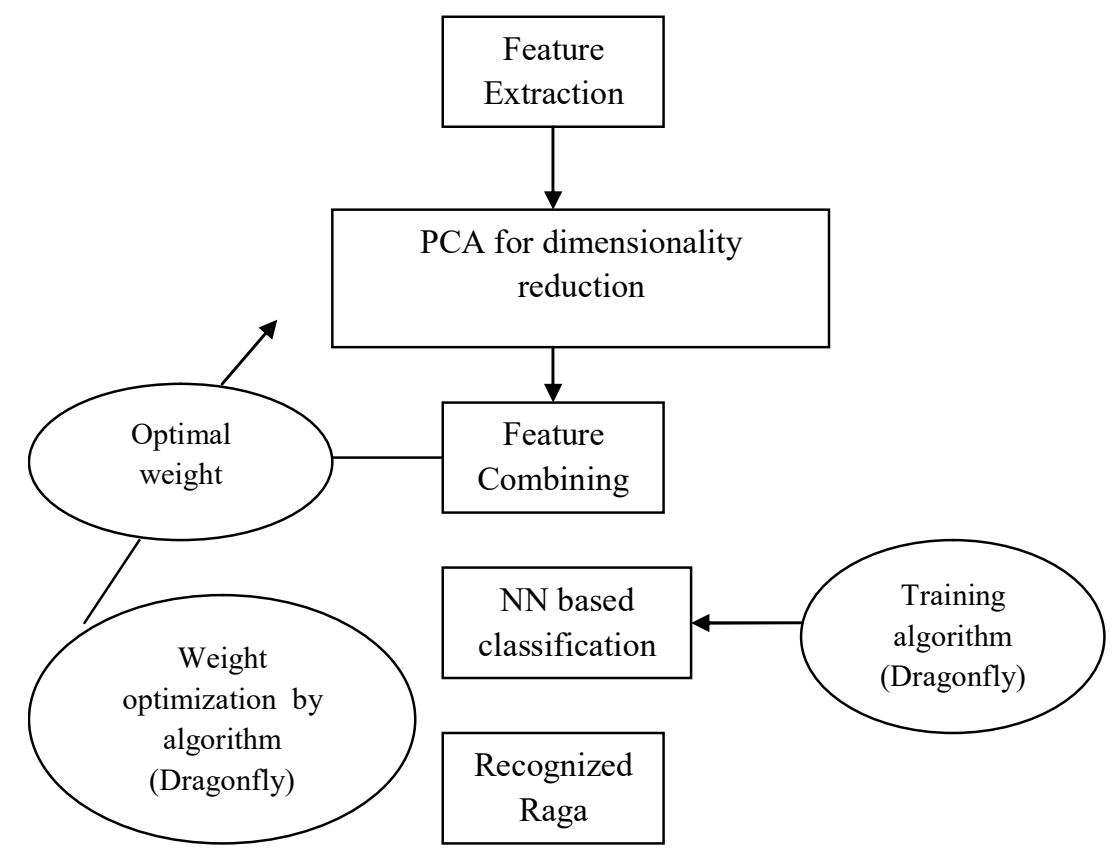

Fig.1. Schematic model of the developed raga recognition

Eq. (21) represents the fitness function of the developed technique, whereas $\left(\mathrm{D}^{\prime}-\mathrm{D}_{\mathrm{i}}^{\prime}\right)$ indicates distance amid a specific feature and remaining features.

$$
\mathrm{O}^{\mathrm{F}}=\sum_{\mathrm{i}=1}^{\mathrm{o}}\left(\mathrm{D}^{\prime}-\mathrm{D}_{\mathrm{i}}^{\prime}\right)
$$

\subsection{Feature Extraction}

From input raga audio signal $y(n), 3$ features namely $\operatorname{NMF}_{f^{\prime}}(\mathrm{n}), \operatorname{STFT}_{\mathrm{f}^{\prime}}(\mathrm{n})$, and $\operatorname{Pitch}_{\mathrm{f}^{\prime}}(\mathrm{n})$ are extracted that is (8), (17), and (18) correspondingly. To reduce the dimensions of the features aforesaid features are given as input to the subsequent procedure.

\subsection{Dimensionality Reduction}

The extracted $\mathrm{NMF}_{\mathrm{f}^{\prime}}(\mathrm{n}) \mathrm{STFT}_{\mathrm{f}^{\prime}}(\mathrm{n})$, and $\operatorname{Pitch}_{\mathrm{f}^{\prime}}(\mathrm{n})$ are fed as input. Initially, from input, a cluster of $\hat{\mathrm{D}}$ data vectors $\mathrm{v}_{1}, \ldots \ldots \ldots \mathrm{v}_{\hat{\mathrm{D}}}$ is chosen and $v^{0}$ indicates a particular group seen of variables $i$. The empirical mean curve estimation is performed by means of all co columns: $c=1, \ldots \ldots . \mathrm{i}$. With $\mathrm{i} \times 1$ dimensions the ensuing mean value is positioned in $\mathrm{l}_{(\mathrm{i})}$, that is stated in eq. (22). As per eq. (23), the deviation of mean is calculated, whereas $J$ indicates $\hat{D} \times i$ matrix and $\mathrm{j}$ is column vector $\hat{\mathrm{D}} \times 1$ of all $1 \mathrm{~s}: \mathrm{j}[\mathrm{v}]=1$

$$
\begin{aligned}
& \mathrm{l}(\mathrm{i})[\mathrm{co}]=\frac{1}{\hat{\mathrm{D}}} \mathrm{R}[\mathrm{v}, \mathrm{co}] \\
& \mathrm{J}=\mathrm{R}-\mathrm{jco}{ }^{\mathrm{T}}
\end{aligned}
$$

As stated in eq. (24), the mathematical formula of the covariance matrix $\mathrm{CV}^{(\mathrm{X})}$ is performed. By valuing the matrix $\mathrm{X}$ both the eigenvalues and eigenvector are calculated, as exhibited in Eq. (25), which also diagonalizes $\mathrm{CV}^{(\mathrm{X})}$.

$$
\begin{aligned}
& \mathrm{CV}^{(\mathrm{X})}=\frac{1}{\hat{\mathrm{D}}-1} \mathrm{~J}^{*} \mathrm{~J} \\
& \mathrm{X}^{-1} \mathrm{CV}(\mathrm{X}) \mathrm{X}=\mathrm{d} \\
& (\mathrm{X})
\end{aligned}
$$

whereas d ${ }^{(X)}$ indicates a diagonal matrix of eigenvalues. The eigenvalue matrix minimization of $\mathrm{d}^{(\mathrm{X})}$ is obtained by column sorting of the eigenvector matrix Y. As exhibited in eq. (26), the cumulative formulation of energy content cu, that holds the summation of the energy content of eigenvalues from one via co. 


$$
\mathrm{cu}[\mathrm{co}]=\sum_{\hat{\mathrm{D}}=1}^{\mathrm{co}} \mathrm{d}(\mathrm{X})[\hat{\mathrm{D}}, \hat{\mathrm{D}}] \text { for } \mathrm{co}=1, \ldots . \mathrm{i}
$$

By storing the $\mathrm{cm}$ column of $\mathrm{X}$ as $\mathrm{Z}$ matrix eigenvectors subset is chosen. The $\mathrm{cm}$ cost is chosen by exploiting e vector. The $\hat{\mathrm{X}}$ matrix column $\hat{\mathrm{X}}=\mathrm{C} \cdot \mathrm{Z}=\mathrm{KLT}\{\mathrm{L}\}$ indicates the vector that ascertains the KLT in L row matrix. In Eq. (27), C matrix is ascertained as stated, whereas $\mathrm{t}^{\prime}=\mathrm{t}^{\prime}(\mathrm{co})=\left\{\sqrt{\mathrm{CV}^{(\mathrm{X})}|\mathrm{co}, \mathrm{co}|}\right\}: \mathrm{co}=1, \ldots . \mathrm{i}$, the procedure consequences in dimensional minimized feature. Here, the PCA process is used individually for all 3 extracted features, which creates dimensional minimized $\operatorname{NMF~NMF}_{f^{\prime}}^{\text {red }}(\mathrm{n}), \operatorname{STFT~STFT}_{\mathrm{f}^{\prime}}^{\text {red }}(\mathrm{n})$, and $\operatorname{Pitch}_{\mathrm{f}^{\prime}}^{\text {red }}(\mathrm{n})$. Eq. (28) states dimension minimized features, which are integrated to form $\mathrm{F}^{\mathrm{red}}(\mathrm{n})$.

$$
\begin{aligned}
& \mathrm{C}=\frac{\mathrm{J}}{\mathrm{j} \cdot \mathrm{t}^{\hat{\mathrm{X}}}} \\
& \mathrm{F}^{\mathrm{red}}(\mathrm{n})=\left\{\operatorname{STFT}_{\mathrm{f}^{\prime}}^{\mathrm{red}}(\mathrm{n}), \mathrm{NMF}_{\mathrm{f}^{\prime}}^{\mathrm{red}}(\mathrm{n}), \operatorname{Pitch}_{\mathrm{f}^{\prime}}^{\mathrm{red}}(\mathrm{n})\right\}
\end{aligned}
$$

\subsection{Raga Recognition}

In this research, NN [10] classifier is exploited to recognize suitable raga. Furthermore, the Dragonfly optimization technique is exploited to train NN. The integrated dimensional minimized feature $\mathrm{F}^{\mathrm{red}}(\mathrm{n})$ is stated as input that is stated in Eq. (29), whereas N indicates the count of features.

$$
\mathrm{F}^{\mathrm{red}}(\mathrm{n})=\left\{\mathrm{f}_{1}, \mathrm{f}_{2}, \ldots \ldots . . \mathrm{f}_{\mathrm{N}}\right\}
$$

Eq. (30), (31), and (32) indicates the network model is indicated, whereas $\mathrm{w}_{(\mathrm{bi})}^{(\mathrm{H})}$ indicates bias weight to $i^{\text {th }}$ hidden neuron, $\mathrm{i}$ signifies hidden neuron, $\mathrm{n}_{\mathrm{i}}$ indicates the count of input neurons, $\mathrm{w}_{(\mathrm{bm})}$ symbolizes output bias weight to $\mathrm{m}^{\text {th }}$ layer, $\mathrm{n}_{\mathrm{h}}$ symbolizes count of hidden neurons, $\mathrm{w}_{(\mathrm{im})}^{(\mathrm{o})}$ symbolizes output weight from $\mathrm{i}^{\text {th }}$ hidden neuron to $\mathrm{m}^{\text {th }}$ layer. Eq. (31), states network output $\hat{\mathrm{g}}_{\mathrm{m}}$ whereas $\mathrm{g}_{\mathrm{m}}$ represents the actual output.

$$
\begin{aligned}
& \mathrm{e}^{(\mathrm{H})}=\mathrm{NF}\left(\mathrm{w}_{(\mathrm{bi})}^{(\mathrm{H})}+\sum_{\mathrm{j}=1}^{\mathrm{n}_{\mathrm{i}}} \mathrm{w}\left(\mathrm{(ji}^{\mathrm{H}}\right) \mathrm{F}^{\mathrm{red}}(\mathrm{n})\right) \\
& \left.\hat{\mathrm{g}}_{\mathrm{m}}=\mathrm{NF}(\mathrm{w}(\mathrm{\textrm {o }}) \mathrm{bm})+\sum_{\mathrm{i}=1}^{\mathrm{n}_{\mathrm{h}}} \mathrm{w}(\mathrm{im}) \mathrm{e}_{\mathrm{i}}^{(\mathrm{H})}\right) \\
& \mathrm{w}^{*}=\underset{\left\{\mathrm{w}(\mathrm{bi}), \mathrm{w}_{(\mathrm{ji})}^{(\mathrm{Hi})}, \underset{(\mathrm{o})}{\arg \min )}, \mathrm{w}_{(\mathrm{im})}^{(\mathrm{o})}\right)}{\mathrm{n}_{\mathrm{o}}} \sum_{\mathrm{m}=1}^{\mathrm{n}_{\mathrm{m}}}-\hat{\mathrm{g}}_{\mathrm{m}}
\end{aligned}
$$

\section{Proposed Dragonfly ALgorithm}

In 2016, Dragonfly algorithm (DA) is developed by Mirjalili [11], which is a recent and interesting natureinspired meta-heuristic optimization approached exploited to resolve a large variety of optimization problems. DA is on the basis of a natural dynamic (migratory) and static (feeding) swarming behaviors of dragonflies. A large number of dragonflies make the swarms migrate in one direction over long distances and distract from enemies in the exploitation phase,. Nevertheless, dragonflies make small groups and fly back and forth over a small area to search for food and attract flying preys in the exploration phase. The swarming behavior of dragonflies is represented by exploiting five operators [11]: Separation: It is a model, which assures to keep search agents evade in the neighborhood from each other and it is exhibited in eq. (33), $\mathrm{S}_{\mathrm{i}}$ indicates Separation.

$$
S_{i}=-\sum_{j=1}^{N} X-X_{i}
$$

Alignment:

It shows the particular search agent velocity is how equivalent with other search agent's velocity in the neighborhood and it is exhibited in eq. (34): $\mathrm{A}_{\mathrm{i}}$ indicates alignment. 


$$
A_{i}=\frac{\sum_{j=1}^{N} v_{j}}{N}
$$

whereas

$$
\mathrm{V}_{\mathrm{j}}
$$

indicates

$\mathrm{j}^{\text {th }}$

neighbor

speed.

Cohesion: It shows how individuals fly from neighborhood area to center of mass, which is exhibited in eq. (35), $\mathrm{C}_{\mathrm{i}}$ indicates cohesion.

$$
\mathrm{C}_{\mathrm{i}}=\frac{\sum_{\mathrm{j}=1}^{\mathrm{N}} \mathrm{x}_{\mathrm{j}}}{\mathrm{N}}-\mathrm{X}
$$

- Attraction: It indicates how the food source attracts individuals which fly towards, is exhibited in Eq. (36), $\mathrm{F}_{\mathrm{i}}$ indicates the movement speed into a food source.

$$
\mathrm{F}_{\mathrm{i}}=\mathrm{F}_{\mathrm{loc}}-\mathrm{X}
$$

whereas

$$
\mathrm{F}_{\text {loc }}
$$

indicates

food

source

location.

- Distraction: It indicates individuals' tendency to fly far from an enemy is exhibited in Eq. (37), $E_{i}$ indicates enemy distribution level.

whereas indicates the enemy's location.

The general model of the Particle Swarm Optimization technique is exploiting by the Dragonfly algorithm as it exploits $\quad 2 \quad$ vectors to update ocation
The step vector is stated in eq. (38), which serves to change the movement of dragonflies'.

$$
\text { Eq. (39) shows an optimal balance among exploration }
$$
161 and exploitation. The inertia weight is computed using Eq. (40).

$$
\begin{aligned}
& \mathrm{s}=2 \times \mathrm{r} \times \mathrm{pct} \\
& \mathrm{a}=2 \times \mathrm{r} \times \mathrm{pct} \\
& \mathrm{c}=2 \times \mathrm{r} \times \mathrm{pct} \\
& \mathrm{f}=2 \times \mathrm{r} \\
& \mathrm{e}=\mathrm{pct} \\
& \mathrm{w}=0.9-\text { iter } * \frac{(0.9-0.4)}{\text { max_iter }}
\end{aligned}
$$

whereas pct is computed as Eq. (40)

$$
p c t=\left\{\begin{array}{l}
0.1-\frac{0.2 \times \text { iter }}{\text { max_iter }}, \text { if }(2 \times \text { iter }) \leq \text { max_iter } \\
0, \quad \text { o.w }
\end{array}\right.
$$

whereas $r$ indicates an arbitrary number in the interval of $[0,1]$. As in Eq. (41), an individual location is updated:

$t$ indicates the current step

$$
\mathrm{X}_{\mathrm{t}+1}=\mathrm{X}_{\mathrm{t}}+\Delta \mathrm{X}_{\mathrm{t}+1}
$$

\section{Result and Discussion}

In this section, the raga recognition technique was performed. Moreover, 10 ragas were exploited such as Chakravaka, Gowrimanohari, Charukeshi, Harikambodi, Kalyani, hemavaca, Shankarabharana, Karaharapriya, Mayamalagowla, and Todicarnatic. For each raga, the number of music data is three and the time duration is minimal $30 \mathrm{sec}$ to maximal 1 minute. In real-time case, the ragas for the present research were attained. Furthermore, threefeatures namely Pitch Nonnegative matrix factorization (NMF), and Short Time Fourier Transform (STFT), were extracted from the signal. Here, the positive and negative metrics were considered, the positive metrics namely accuracy, precision, specificity, NPV, sensitivity, F1score and Matthews correlation coefficient (MCC) and negative metrics namely False Negative Rate (FNR), False Positive Rate (FPR), and False Discovery Rate (FDR). 
Fig 2 exhibits the analysis of the developed model over existing classifiers regarding the STFT feature. Here, the proposed method is $40 \%$ better than the KNN, and $41 \%$ better than the SVM in terms of the accuracy. Fig 3 demonstrates the analysis of the developed model over existing classifiers concerning the NMF feature. Here, the proposed method is $40 \%$ better than the KNN, and $21 \%$ better than the SVM in terms of the accuracy. Fig 4 illustrates the analysis of the developed model over existing classifiers regarding the pitch feature. Here, the performance outcomes have shown the betterment of development technique in recognizing suitable raga even the singers is diverse. Here, the proposed method is $20 \%$ better than the KNN, and 4\% better than the SVM in terms of the accuracy.

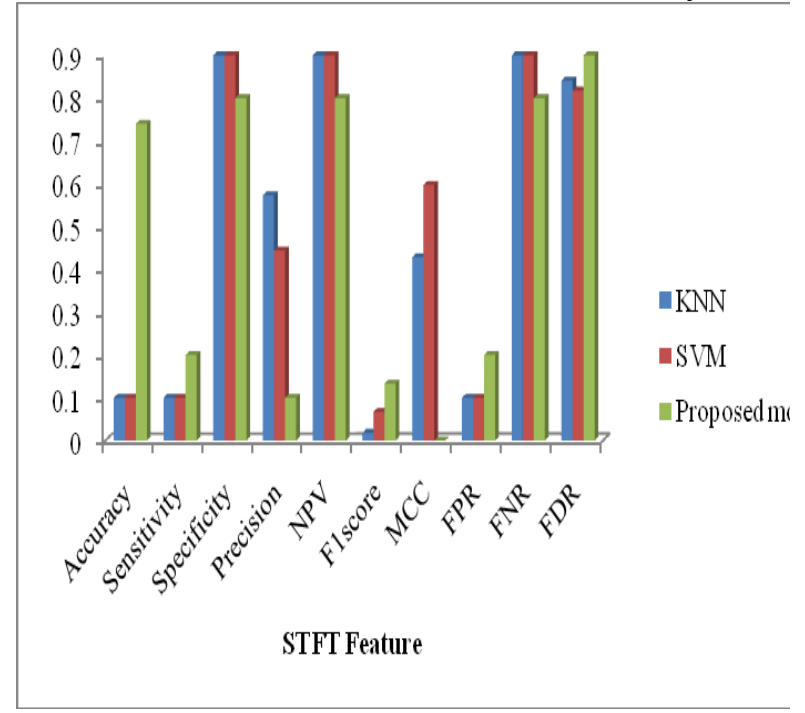

Fig 2: performance analysis of STFT Feature for raga classification

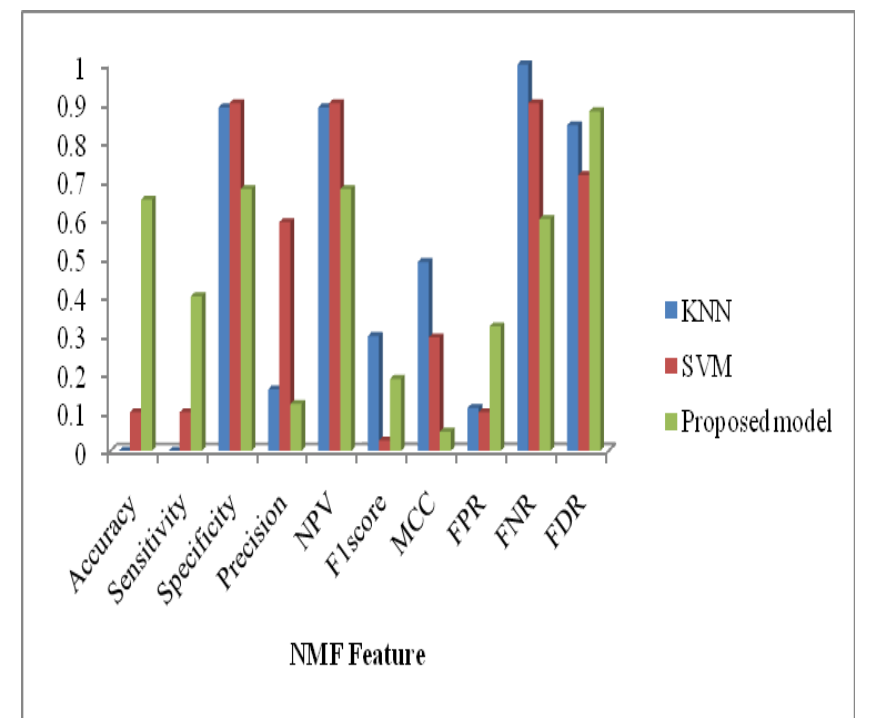

Fig 3: performance analysis of NMF Feature for raga classification 


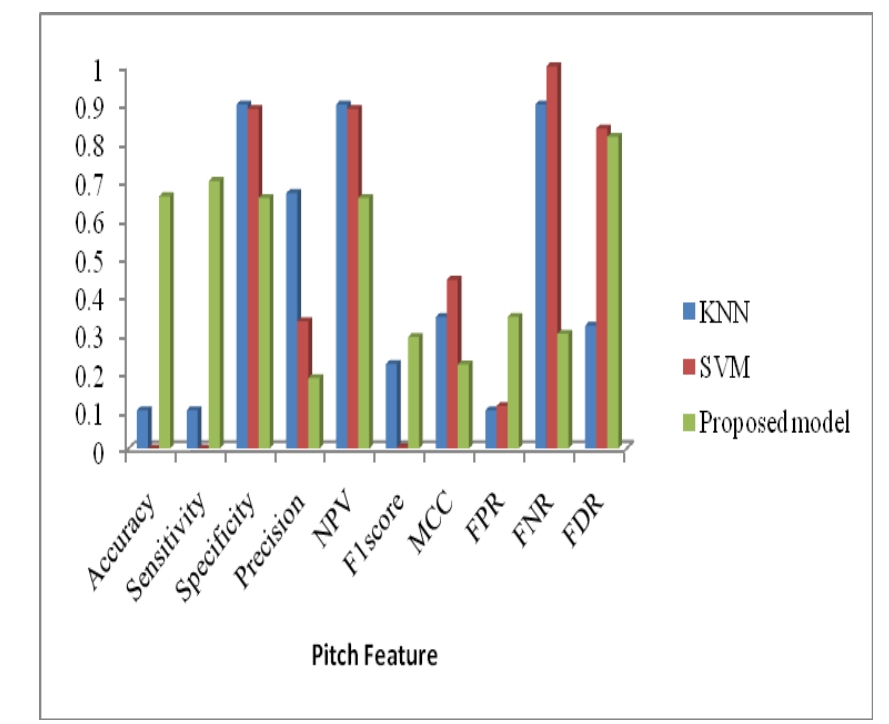

Fig 4: performance analysis of pitch Feature for raga classification

\section{Conclusion}

A raga recognition technique was presented to identify the raga in an accurate way; therefore Carnatic genre of music was optimally identified in this research. Furthermore, an adaptive NN classifier was presented, in that, a well-known Dragonfly optimization technique was used to train the NN technique. The developed technique has attained its uniqueness by augmenting optimal weight with the input feature set of the Neural network technique. Finally, the developed technique was evaluated to traditional models and from consequences it was analyzed that precise of the developed technique. Therefore, the entire analysis has shown the superiority of the developed technique in identifying suitable raga.

\section{References}

[1] M. S. SinithShikha TripathiK. V. V. Murthy,"Raga recognition using fibonacci series based pitch distribution in Indian Classical Music", Applied Acoustics, 30 April 2020.

[2] Prafulla KalapatapuSrihita GoliAruna Malapati,"A Study on Feature Selection and Classification Techniques of Indian Music",Procedia Computer Science, 2016.

[3] Banriskhem K. KhonglahS. R. Mahadeva Prasanna,"Speech / music classification using speech-specific features", Digital Signal Processing, January 2016.

[4] Snehlata BardeVeena Kaimal,"Chapter 5: Speech recognition technique for identification of raga", Cognitive Informatics, Computer Modelling, and Cognitive Science, 17 April 2020.

[5] Y. V. Srinivasa MurthyShashidhar G. Koolagudi,"Classification of vocal and non-vocal segments in audio clips using genetic algorithm based feature selection (GAFS)", Expert Systems with Applications, 7 April 2018.

[6] Lütfiye Durak and Orhan Arıkan," Short-Time Fourier Transform: Two Fundamental Properties and an Optimal Implementation", IEEE TRANSACTIONS ON SIGNAL PROCESSING, vol. 51, no.5, pp. 1231-1241, may 2003.

[7] Lijun ZHANG, Zhengguang CHEN, Miao ZHENG and Xiaofei HE, "Robust non-negative matrix factorization", Front. Electr. Electron. Eng, vol. 6, no. 2, pp. 192-200, 2011

[8] Camacho A and Harris JG, " A sawtooth waveform inspired pitch estimator for speech and music.", J Acoust Soc Am., vol. 124, no. 3, pp. 1638-52, 2008.

[9] Debora C. Correa and Francisco Ap. Rodrigues, "A survey on symbolic data-based music genre classification", Expert Systems with Applications, vol.60, pp. 190-210, October 2016.

[10] Y. Mohan, S. S. Chee, D. K. P. Xin and L. P. Foong, "Artificial neural network for classification of depressive and normal in EEG," IEEE EMBS Conference on Biomedical Engineering and Sciences (IECBES), Kuala Lumpur , pp. 286-290, 2016.

[11] Surya Narayana SankaTirumala Reddy YarramJ. R. K. Kumar Dabbakuti,"Dragonfly algorithm based spectrum assignment for cognitive radio networks", Materials Today: ProceedingsAvailable online 6 January 2021.

[12] Amol V Dhumane,"Examining User Experience of eLearning Systems using EKhool Learners", vol. 3, no 4, October 2020.

[13] Quazi M. H and Dr. S. G. Kahalekar,"Adaptive filtering in EEG Signal for Artifacts Removal using Learning Algorithm", Journal of Networking and Communication Systems,vol. 2, no 2, April 2019. 